\title{
Aspects of semilocal BPS vortex in systems with Lorentz symmetry breaking
}

\author{
C. H. Coronado Villalobos ${ }^{1, \mathrm{a}}$, J. M. Hoff da Silva ${ }^{1, \mathrm{~b}}$, M. B. Hott ${ }^{1, \mathrm{c}}$, H. Belich ${ }^{2, \mathrm{~d}}$ \\ ${ }^{1}$ Departamento de Física e Química, UNESP, Univ Estadual Paulista, Av. Dr. Ariberto Pereira da Cunha, 333, Guaratinguetá, SP, Brazil \\ ${ }^{2}$ Departamento de Física e Química, Universidade Federal do Espí rito Santo (UFES), Av. Fernando Ferrari, 514, Vitória, ES 29060-900, Brazil
}

Received: 30 November 2013 / Accepted: 25 February 2014 / Published online: 11 March 2014

(C) The Author(s) 2014. This article is published with open access at Springerlink.com

\begin{abstract}
The existence is shown of a static self-dual semilocal vortex configuration for the Maxwell-Higgs system with a Lorentz-violating CPT-even term. The dependence of the vorticity upper limit on the Lorentz-symmetrybreaking term is also investigated.
\end{abstract}

\section{Introduction}

The Standard Model (SM) has recently passed its final test. The discovery of the Higgs boson has confirmed the last prediction of a model of undisputed success. Despite the tremendous success of this model, it presents a description of massless neutrinos and cannot incorporate gravity as a fundamental interaction.

We expect that new physics may appear if we reach the $\mathrm{TeV}$ scale and beyond. But if General Relativity and SM are effective theories, what could be guide concepts to obtain physics beyond SM? The Higgs mechanism is a fundamental ingredient, used in the electroweak unification, to obtain the properties of low-energies physics. The breaking of a symmetry by a scalar field describing a phase transition is currently used in many branches of sciences. Without going into details, we would say that at the microscopic level an effective field generated spontaneously can give clues on how to get the fundamental theory. In relativistic systems, the field that realizes the breaking must be a scalar in order to preserve Lorentz symmetry.

In nonrelativistic quantum systems, phase transitions such as in ferromagnetic systems, the rotation symmetry is broken due to the influence of a magnetic field. For relativistic systems, the realization of symmetry breaking can be extended

\footnotetext{
a e-mail: ccoronado@feg.unesp.br

b e-mail: hoff@feg.unesp.br

c e-mail: hott@feg.unesp.br

de-mail: belichjr@gmail.com
}

by considering a background given by a constant 4 -vector field that breaks the symmetry $S O(1,3)$ and no longer the symmetry $S O(3)$. This new possibility of spontaneous violation was first suggested by Kostelecky and Samuel [1] in 1989 , indicating that, in the string field theory scenario, the spontaneous violation of symmetry by a scalar field could be extended to other classes of tensor fields.

This line of research including spontaneous violation of the Lorentz symmetry in the Standard Model is known in the literature as Standard Model Extension (SME) [2-12], and the breaking is implemented by condensation of tensors of rank $>1$. This program includes investigations over all the sectors of the standard model-fermion, gauge, and Higgs sectors (a very incomplete list includes [13-20])—as well as gravity extensions [21]. Following this reasoning, the study of topological defects has also entering this framework [2226]. Quite recently [27], it was demonstrated that a MaxwellHiggs systems with a CPT-even Lorentz symmetry-violating term yields Bogomol'nyi-Prasad-Sommerfield (BPS) [28, 29] vortex solutions enjoying fractional quantization of the magnetic field.

Topological defects arising from spontaneous symmetry breaking are physical systems of interest in a wide range of theories, from condensed matter to cosmology [30-32]. These defects may arise from an abelian, as well as nonabelian, spontaneously broken symmetry. The type of the defect depends on the broken symmetry. Among the typical interesting defects, vortex solutions are a relevant class and their characteristics have been extensively investigated in the literature [33]. So, an interesting program would be to investigate topological defects in a scenario with the violation of Lorentz symmetry and to identify all those quantities which can be directly affected by this special type of breaking, namely Lorentz-symmetry breaking.

One of the benchmarks of the vortex theory is the semilocal vortex [34]. Usually, most part of the study of vortex was restricted to the local symmetry. However, the inclusion of 
a global symmetry, besides the usual local one, may lead to some interesting characteristics in the resulting topological defect as the presence of topological vortex even if the vacuum manifold is simply connected, the presence of infinite defects, and the fact that semilocal strings may end in a cloud of energy.

This paper is partially concerned with the demonstration that semilocal vortices may be found in a usual MaxwellHiggs system plus a CPT-even Lorentz symmetry-violating term. In other words, it is possible to combine the generalized vortex solutions found in [27] and the semilocal structure (Sect. 2). As is well known from the standard properties of the semilocal setup, the minimum of the potential is a three-sphere, which is simply connected. In fact, starting from a $S U(2)_{\text {global }} \otimes U(1)_{\text {local }}$ symmetry, the symmetry breaks down to $U(1)_{\text {local }}$. Hence, the first homotopy group is trivial, i.e., $\pi_{1}\left(S U(2)_{\text {global }} \otimes U(1)_{\text {local }} / U(1)_{\text {local }}\right)=1$. However, the local symmetry also plays its role. At each point on the three-sphere the local symmetry engenders a circle. In this vein, looking at the local symmetry, one realizes that it is possible to obtain infinitely many vortex solutions, corresponding to the breaking of the local symmetry $\left(\pi_{1}\left(U(1)_{\text {local }} / 1\right)=\mathbb{Z}\right)$. Since the potential we shall deal with goes as usual, it is possible to say that as in the usual Higgs-Maxwell case [34], when no Lorentz-violating term is present, the arguments in favor of stable vortices are strong, but not exhaustive. In order to guarantee the existence of semilocal vortices in the Maxwell-Higgs plus Lorentzviolating model, we have to construct the solutions.

It was shown [27] that the presence of the Lorentz symmetry-violating term may lead to a peculiar effect in the vortex size. Hence, in view of the aforementioned characteristic of the semilocal vortex, the solution combining both effects may result in a most malleable defect structure, which is shown to be the case. Besides, we show that the Bradlow limit $[35,36]$ depends on the magnitude of a parameter related to the Lorentz-breaking term, i.e., the vorticity is also affected. In fact, the vorticity increases as the Lorentzviolating term becomes more relevant.

\section{Semilocal vortex with a Lorentz symmetry-breaking term}

We start from the lagrangian density

$$
\begin{aligned}
\mathcal{L}= & -\frac{1}{4} F_{\mu \nu} F^{\mu \nu}-\frac{1}{4}\left(\kappa_{F}\right)_{\mu \gamma \alpha \beta} F^{\mu \gamma} F^{\alpha \beta}+\left|D_{\mu} \Phi\right|^{2} \\
& -\frac{\lambda^{2}}{4}\left(\eta^{2}-|\Phi|^{2}\right)^{2},
\end{aligned}
$$

where $\Phi$ is given by the $S U(2)$ doublet $\Phi^{T}=(\phi \psi)$. The covariant derivative is given by $D_{\mu}=\partial_{\mu}-i e A_{\mu}$ and $F_{\mu \nu}$ is the usual electromagnetic field strength, in such a way that the above lagrangian is endowed with the $S U(2)_{\text {global }} \otimes U(1)_{\text {local }}$ symmetry. Note that it is similar to the lagrangian investigated in [27], except for the presence of the global symmetry.

The $\left(\kappa_{F}\right)_{\mu \gamma \alpha \beta}$ term is the CPT-even tensor. It has the same symmetries as the Riemann tensor, plus a constraint coming from double null trace $\left(\kappa_{F}\right)_{\alpha \beta}^{\alpha \beta}=0$. It may be defined according to

$\left(\kappa_{F}\right)^{\mu \gamma \alpha \beta}=\frac{1}{2}\left(\eta^{\mu \alpha} \kappa^{\gamma \beta}-\eta^{\gamma \alpha} \kappa^{\mu \beta}+\eta^{\gamma \beta} \kappa^{\mu \alpha}-\eta^{\mu \beta} \kappa^{\gamma \alpha}\right)$,

from which it is readily verified that

$\left(\kappa_{F}\right)_{\mu \gamma \alpha \beta} F^{\mu \gamma} F^{\alpha \beta}=2 \kappa_{\mu \beta} F_{\alpha}^{\mu} F^{\alpha \beta}$.

As we want to generalize the uncharged vortex solution, it is necessary to set $\kappa_{0 i}=0$, since from the stationary Gauss law obtained from (1) this last condition decouples the electric and magnetic sectors. Hence, considering the temporal gauge $A_{0}=0$, the energy functional is given by

$$
\begin{aligned}
E= & \int \mathrm{d}^{2} x\left[\frac{1}{2}\left[\left(1-\operatorname{tr}\left(\kappa_{i j}\right)\right) \delta_{a b}+\kappa_{a b}\right] B_{a} B_{b}+|\vec{D} \Phi|^{2}\right. \\
& \left.+\frac{\lambda^{2}}{4}\left(\eta^{2}-|\Phi|^{2}\right)^{2}\right] .
\end{aligned}
$$

By working with cylindrical coordinates from now on, we implement the standard vortex ansatz,

$$
\begin{aligned}
\phi & =\eta g_{1}(r) e^{i n \theta}, \\
\psi & =\eta g_{2}(r) e^{i n \theta_{2}}, \\
A_{\theta} & =-\frac{1}{e r}[a(r)-n] .
\end{aligned}
$$

The functions $g_{1}(r), g_{2}(r)$, are regular functions and in the case of a typical vortex solution they have no dynamics as $r \rightarrow \infty$. It is quite enough to ensure that the coupling of the fields to the gauge field leads to the phase correlation $\theta_{2}=\theta+c, c$ being a constant. Obviously, for a typical vortex solution we shall have the following boundary conditions for $a(r)$ :

$a(r) \rightarrow n$ as $r \rightarrow 0$ and $a(r) \rightarrow 0$ as $r \rightarrow \infty$.

With the chosen ansatz, the magnetic field is trivially given by

$B_{z} \equiv B=-\frac{1}{e r} \frac{\mathrm{d} a}{\mathrm{~d} r}$ 
Now, taking $\kappa_{11}+\kappa_{22}=s$ it is possible to write

$$
\begin{aligned}
E= & \int \mathrm{d}^{2} r\left[\frac{1}{2}(1-s) \frac{1}{e^{2} r^{2}}\left(\frac{\mathrm{d} a}{\mathrm{~d} r}\right)^{2}+\eta^{2}\left(\frac{\mathrm{d} g_{1}}{\mathrm{~d} r}\right)^{2}\right. \\
& +\eta^{2}\left(\frac{\mathrm{d} g_{2}}{\mathrm{~d} r}\right)^{2}+\frac{a^{2} \eta^{2}}{r^{2}}\left(g_{1}^{2}+g_{2}^{2}\right) \\
& \left.+\frac{\eta^{4} \lambda^{2}}{4}\left(1-g_{1}^{2}-g_{2}^{2}\right)^{2}\right] .
\end{aligned}
$$

By imposing the self-duality condition [27] $\lambda^{2}=2 e^{2} /(1-$ $s)$ - the equivalent to the equality of the scalar and gauge field masses - it is possible to rearrange the terms in (8) after a bit of algebra, such as

$$
\begin{aligned}
E= & \int \mathrm{d}^{2} r\left[\frac{(1-s)}{2}\left(\frac{1}{e r} \frac{\mathrm{d} a}{\mathrm{~d} r} \pm \frac{e \eta^{2}}{(1-s)}\left(1-g_{1}^{2}-g_{2}^{2}\right)\right)^{2}\right. \\
& +\eta^{2}\left(\mp \frac{a g_{1}}{r}+\frac{\mathrm{d} g_{1}}{\mathrm{~d} r}\right)^{2}+\eta^{2}\left(\mp \frac{a g_{2}}{r}+\frac{\mathrm{d} g_{2}}{\mathrm{~d} r}\right)^{2} \\
& \left. \pm \frac{\eta^{2}}{r}\left(\frac{\mathrm{d}\left(a g_{1}^{2}\right)}{\mathrm{d} r}\right) \pm \frac{\eta^{2}}{r}\left(\frac{\mathrm{d}\left(a g_{2}^{2}\right)}{\mathrm{d} r}\right) \mp \frac{\eta^{2}}{r} \frac{\mathrm{d} a}{\mathrm{~d} r}\right]
\end{aligned}
$$

In the above expression, the linear terms are those which contribute to the minimum energy when the self-dual equations are fulfilled. The first order BPS equations are given by

$\frac{\mathrm{d} g_{1}}{\mathrm{~d} r}= \pm \frac{g_{1} a}{r}, \frac{\mathrm{d} g_{2}}{\mathrm{~d} r}= \pm \frac{g_{2} a}{r}$

and

$-\frac{1}{e r} \frac{\mathrm{d} a}{\mathrm{~d} r}= \pm \frac{e \eta^{2}}{(1-s)}\left(1-g_{1}^{2}-g_{2}^{2}\right)$

while the energy minimum is given by

$E_{\min }= \pm 2 \pi \eta^{2} n\left(1-g_{1}^{2}(0)-g_{2}^{2}(0)\right)$,

where in the last equation we have used the boundary conditions (6) and the fact that $g_{1}$ and $g_{2}$ are regular functions.

Now we are in a position to show that despite the fact that the vacuum manifold is simply connected the field configuration vanishing at the center of the vortex is compatible with the above framework. Introducing $g^{2}=g_{1}^{2}+g_{2}^{2}$, subject to the boundary condition $g \rightarrow 0$ as $r \rightarrow 0$, one immediately gets

$E_{\min }= \pm 2 \pi \eta^{2} n=\eta^{2} e\left|\Phi_{B}\right|$

where $\Phi_{B}$ is the magnetic flux, and

$B=-\frac{1}{e r} \frac{\mathrm{d} a}{\mathrm{~d} r}= \pm \frac{e \eta^{2}}{(1-s)}\left(1-g^{2}\right)$

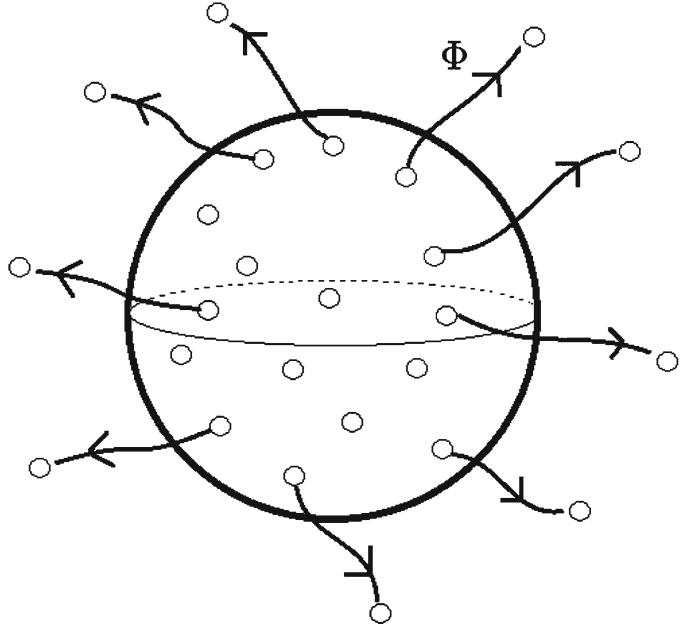

Fig. 1 The vacuum manifold: the infinitely many possibilities of spontaneous local symmetry breaking and the mapping in vortex configurations

The two remaining equations may be bound together as

$\frac{\mathrm{d} g}{\mathrm{~d} r}= \pm \frac{g a}{r}$

Equations (14) and (15) are identical to the self-dual equations found in [27]. Therefore the same conclusions obtained there are applicable to the present semilocal case. Of particular interest, their numerical results attest to the stability of the BPS vortex solutions.

At this point it would be interesting to say a few words concerning the semilocal solutions. From the first order equations (10) and (15), it is easy to see that

$\frac{1}{g} \frac{\mathrm{d} g}{\mathrm{~d} r}=\frac{1}{g_{i}} \frac{\mathrm{d} g_{i}}{\mathrm{~d} r}$,

where $i=1,2$. Hence the solutions shall obey $g \sim g_{i}$ and by the constraint $g^{2}=g_{1}^{2}+g_{2}^{2}$ we have $1=f_{1}^{2}+f_{2}^{2}$, where the $f_{i}$ are numerical factors. Thus, we see that there are plenty configurations satisfying the boundary conditions. Each of this configurations corresponds to local spontaneous symmetry breaking of the vacuum manifold. In fact, the vacuum manifold associated to the $S U(2)_{\text {global }} \times U(1)_{\text {local }}$ symmetry may be understood as a three-sphere of which each point (due to the local symmetry) is given by a $S^{1}$ circle. It is nothing but the fiber bundle formulation of the vacuum, being the base space that one associates to the $S U(2)_{\text {global }}$ (the three-sphere) and the typical fiber performed by the manifold associated to the $U(1)_{\text {local }}\left(S^{1}\right.$ circles). The projections are global transformations while the fiber is a gauge transformation. A particular solution of $\left(1=f_{1}^{2}+f_{2}^{2}\right)$ means a given $S^{1} \rightarrow S^{1}$ mapping performed by $\Phi$. The infinitely many possibilities evinced by the equation $1=f_{1}^{2}+f_{2}^{2}$ stands for the infinite possibilities of local symmetry breaking; see Fig. 1. Finally, the situa- 
tion from the vacuum manifold is clear: the local symmetry breaking lead to special vortex configurations which can end since the base manifold is simply connected.

\section{3 s parameter and Bradlow limit}

It was demonstrated in [27] that the Lorentz-violating parameter $s$ plays an important role acting as an element able to control both the radial extension and the amplitude of the defect. In summary, the larger the $s$ parameter, the more compact is the vortex in the sense that the scalar field reaches its vacuum value (or, equivalently, the gauge field goes to zero) in a reduced radial distance in comparison with the situation when the Lorentz symmetry is preserved, the so-called Abrikosov-Nielsen-Olesen vortices. Thus, if one applies this model to the scenario of type-II superconductors one sees that the Lorentz symmetry-breaking term is responsible to enhancing of the superconducting phase.

It is instructive to relate this effect with the maximum vorticity which a noninteracting static vortex system may acquire in a given compact base manifold of area $\mathcal{A}$. This upper bound on the vorticity is the so-called Bradlow limit [35]. Integrating over Eq. (14) and choosing positive vorticity, it is easy to see that

$\frac{1}{e} \int_{0}^{2 \pi} \mathrm{d} \theta \int_{0}^{\infty} r \mathrm{~d} r \frac{1}{r} \frac{\mathrm{d} a}{\mathrm{~d} r}=\int \mathrm{d}^{2} r \frac{e \eta^{2}}{1-s}\left(1-g^{2}\right)$,

and then

$n \leq \frac{e^{2} \eta^{2}}{2 \pi(1-s)} \mathcal{A}$.

Note that for $s=0$ the usual Bradlow limit is recovered, as expected. As $s$ grows, however, so does the upper limit. In other words it is possible to saturate the manifold with more vorticity.

If we contrast this situation with the information that as $s$ grows the vortices become more compact, we see that these two effects are related: the more increasing $s$, the more compact the vortex. The more compact the vortex, the more vortices with vorticity one are allowed within the same base manifold. This may be regarded as growth in the number of vortices shown in a condensed matter vortex sample under an external (fixed direction) magnetic field, or as reduction of the vortex core size due to an increase in the rotation frequency of an electrically neutral superfluid.

Finally, as $s$ approaches 1 it is possible to see that the Bradlow limit blows up. Again, it is in consonance with the analysis performed in [27], where this limit means an extremely short-range theory in which the vortex core length goes to zero but the intensity of the magnetic field inside the vortex increases. Similarly to what happens in type IIsuperconductors, a phase transition where the multiplicity of vortices with vorticity one is favored rather than the melting of the condensate might occur. Such behavior of the magnetic field reinforcing the superconducting phase occurs in ferromagnetic materials that have ferromagnetism coexisting with superconductivity [37]. Parenthetically, if one wants to be in touch with quantum field theory bounds, we notice that the bounds $s \in(-1, \sqrt{2}-1)$ can be obtained by comparison with the results found in the detailed study carried out in [38] for the bounds on the parameter in order to guarantee not only the causality and the unitarity in the dynamic regime, but also the stability of vortex-like configurations (stationary regime) in the Abelian-Higgs model with the CPT-even Lorentz symmetry term in the electromagnetic sector. If we are interested in preserving causality but relaxing the unitarity of the model, we have to take into account the whole interval $s \in(-1,1)$ (we obtain this domain using the results of Ref. [38] which was adapted to our case). In verifying possible instabilities, such as those resulting in phase transitions, one has to consider the values of $s$ in this range. As $s$ approaches 1 it is possible to see that the Bradlow limit blows up, signalizing a phase transition.

On the other hand, it is expected that the spontaneous violation of Lorentz symmetry occurs at high-energy (Grand Unification or Planck scale), while at our energy scale it is manifested only very weakly. In [39] the same violating term as used in our article is investigated with $0<s \ll 1$. In fact, reference [40] presents a table with possible values of Lorentz-symmetry-breaking parameters for a wide class of violating sources in the context of the SME. By considering the results presented in [40-42] when the even sector of the SME is taken into account we conclude that $|s|<10^{-14}$. Then, for those allowed values of $s$, we cannot see this transition, once the phase transition might occur for $s \rightarrow 1$.

\section{Final remarks}

The existence was shown of semilocal BPS vortices in the Maxwell-Higgs model with a Lorentz-symmetry-breaking CPT-even term. The model has, initially, $S U(2)_{\text {global }} \otimes$ $U(1)_{\text {local }}$ and it was demonstrated that minimum energy configurations are found when the scalar field doublet vanishes at the center of the core, even its vacuum manifold being simply connected. As in [34], the vacuum manifold may be understood as a three-sphere pierced by $S^{1}$ circles at each point. Hence, there are infinitely many vortices appearing in the local breaking $U(1)_{\text {local }} \rightarrow 1$. These configurations correspond to the (also infinitely many) possibilities that $g$ may achieve its boundary conditions (remember that $g^{2}=g_{1}^{2}+g_{2}^{2}$ ). Going further we studied the effects of the 
Lorentz-symmetry-breaking term on the vorticity, relating it with the analysis performed for the usual vortex solution in this type of system [27].

We would like to remark that, although the gauge structure of the model still retain $S U_{\text {global }}(2) \otimes U_{\text {local }}(1)$ invariance, it is not evident that the modification of the gauge field kinetic term not necessarily can be always made without spoiling the solution achievement. Particularly, in the case treated here we have resort to the constraint $g^{2}=g_{1}^{2}+g_{2}^{2}$ and to simple algebraic procedures to reach Eq. (15) from (10). The point to be stressed is that the gauge field information, encoded in $a(r)$, must be the same for both parts of the scalar doublet, otherwise the solution cannot be reached. Moreover, among all the possibilities brought about by the Lorentz-symmetrybreaking term, the interesting one, which does not jeopardize the formal construction of the stable BPS solutions, is given when $\kappa_{0 i}$ vanishes, leading to the functional form of the energy as in (8). It turns out that, after all, this possibility appears to be appealing, since it possesses quantum field theory boundaries on its magnitude and, as investigated, leads to an interesting shift in the Bradlow limit, which can be physically interpretable.

It may be instructive to point out a counter example. Suppose a Lagrangian whose Maxwell kinetic term is present, but with another gauge field ruled by an Abelian ChernSimons term as well (the gauge potential being, then, a simple sum of the Maxwell and Chern-Simons standard potentials). The mathematical structure of the action is the same $S U_{\text {global }}(2) \otimes U_{\text {local }}(1)$. Therefore, everything would go as usual. However, if one retains the same scalar field potential it is not possible to achieve a solution via the binding procedure, and one would not have semilocal vortices.

Usually, the search for stable vortex solutions is restricted to modifications of the scalar potential when the gauge field sector of the model is modified. Within this context, every modification leading to an explicit vortex solution, as well as its semilocal generalization, deserves attention. We believe that the modifications (even preserving the mathematical form) on the gauge side of the symmetry are to be treated on the same footing as those in the potential sector, and the solutions must be explicitly constructed and studied.

Acknowledgments The authors thank CAPES and CNPq for the financial support. JMHS thanks to Aristeu Lima for insightful conversations, and the Niels Bohr Institute where this work was partially done.

Open Access This article is distributed under the terms of the Creative Commons Attribution License which permits any use, distribution, and reproduction in any medium, provided the original author(s) and the source are credited.

Funded by $\mathrm{SCOAP}^{3}$ / License Version CC BY 4.0.

\section{References}

1. V.A. Kostelecky, S. Samuel, Phys. Rev. Lett. 63, 224 (1989)

2. D. Colladay, V.A. Kostelecky, Phys. Rev. D 55, 6760 (1997)

3. D. Colladay, V.A. Kostelecky, Phys. Rev. D 58, 116002 (1998)

4. K. Bakke et al., Eur. Phys. J. Plus 127, 102 (2012)

5. K. Bakke et al., J. Phys. G Nucl. Part. Phys. 39, 085001 (2012)

6. K. Bakke et al., J. Phys. G Nucl. Part. Phys. 40, 065002 (2013)

7. K. Bakke et al., Ann. Phys. 333, 272 (2013)

8. R. Casana et al., Phys. Lett. B 718, 620 (2012)

9. R. Casana et al., Phys. Rev. D 86, 125033 (2012)

10. R. Casana et al., Phys. Lett. B 726, 488 (2013)

11. R. Casana et al., Phys. Lett. B 726, 815 (2013)

12. R. Casana et al., Phys. Rev. D 87, 047701 (2013)

13. S.M. Carroll, G.B. Field, R. Jackiw, Phys. Rev. D 41, 1231 (1990)

14. V.A. Kostelecky, M. Mewes, Phys. Rev. Lett. 87, 251304 (2001)

15. D. Colladay, V.A. Kostelecky, Phys. Lett. B 511, 209 (2001)

16. V.A. Kostelecky, C.D. Lane, J. Math. Phys. 40, 6245 (1999)

17. M. Gomes, J.R. Nascimento, AYu. Petrov, A.J. da Silva, Phys. Rev. D 81, 045018 (2010)

18. F.A. Brito, E. Passos, P.V. Santos, Europhys. Lett. 95, 51001 (2011)

19. K. Bakke, H. Belich, E.O. Silva, J. Math. Phys. 52, 063505 (2011)

20. J.L. Boldo, J.A. Helayel-Neto, L.M. de Moraes, C.A.G. Sasaki, V.J.V. Otoya, Phys. Lett. B 689, 112 (2010)

21. V.A. Kostelecky, J.D. Tasson, Phys. Rev. D 83, 016013 (2011)

22. M.D. Seifert, Phys. Rev. D 82, 125015 (2010)

23. A. de Souza Dutra, M. Hott, F.A. Barone, Phys. Rev. D 74, 085030 (2006)

24. D. Bazeia, M.M. Ferreira Jr, A.R. Gomes, R. Menezes, Phys. D (Amsterdam) 239, 942 (2010)

25. A. de Souza Dutra, R.A.C. Correa, Phys. Rev. D 83, 105007 (2011)

26. A.P. Baeta Scarpelli, J.A. Helayel-Neto, Phys. Rev. D 73, 105020 (2006)

27. C. Miller, R. Casana, M.M. Ferreira Jr, E. da Hora, Phys. Rev. D 86, 065011 (2012)

28. E.B. Bogomol'nyi, Sov. J. Nucl. Phys. 24, 449 (1976)

29. M.K. Prasad, C.M. Sommerfield, Phys. Rev. Lett. 35, 760 (1975)

30. M.I. Monastyrsky, Topology of Gauge Fields and Condensed Matter (Plenum Press, New York, 1993)

31. A. Vilenkin, E.P.S. Shellard, Cosmic Strings and Other Topological Defects (Cambridge University Press, Cambridge, 2000)

32. T. Vachaspati, Kinks and Domain Walls: An Introduction to Classical and Quantum Solitons (Cambridge University Press, Cambridge, 2006)

33. A. Vilenkin, Phys. Rep. 121, 263 (1985)

34. T. Vachaspati, A. Achúcarro, Phys. Rev. D 44, 3067 (1991)

35. S.B. Bradlow, Commun. Math. Phys. 135, 1 (1990)

36. G.V. Dunne, Aspects of Chern-Simons Theory, Lectures at the 1998 Les Houches Summer School: Topological Aspects of Low Dimensional Systems [arXiv:hep-th/9902115]

37. D.V. Shopova, D.I. Uzunov, Phys. Lett. A 313, 139 (2003)

38. H. Belich, F.J.L. Leal, H.L.C. Louzada, M.T.D. Orlando, Phys. Rev. D 86, 125037 (2012)

39. G. Betschart, E. Kant, F.R. Klinkhamer, Nucl. Phys. B 815, 198 (2009)

40. V.A. Kostelecky, Neil Russell, Rev. Mod. Phys. 83, 11 (2011)

41. R. Casana et al., Phys. Rev. D 87, 047701 (2013)

42. A.G. de Lima et al., Eur. Phys. J. Plus 128, 154 (2013) 